\title{
VPN Enterprise Network Construction Based on Security
}

\author{
Jinyi Zhou \\ Sichuan Information Technology College, China \\ 30940350@qq.com
}

Keywords: VPN technology; Enterprise; Security network.

\begin{abstract}
It first introduces widespread emergence of network the branches increased and security issues during the enterprise development and internationalization process. To use the VPN technology for network construction based on a comprehensive analysis the enterprise network system requirements and security requirements. It designed commercial IPsec VPN network security based on third layer (network layer), and IPsec security system construction structure. It formed enterprises. In reduces the cost. At the same time, it effectively improves the convenience and safety of enterprise network security.
\end{abstract}

\section{Introduction}

At present, the enterprise has been widely used the Internet information transmission. But because of the high degree of opening, it leads to poor security level, and not conducive to the transmission of confidential corporate high level data. The network structure of the enterprise is usually composed of a network center, several branches, number of partners and mobile remote users (DIAL). There are various connections between the parts, such as, remote dial-up, line, Internet, etc. It constitutes the enterprise network system including information attack threat, the threat of attack system, user attack threat and attack system resources threats. It includes a variety of potential security threats ${ }^{[1]}$. Therefore, to build the enterprise network security effectively is very necessary. In the traditional network architecture, connecting the two private networks is used private line. Private line is an independent closed circuit. It can maintain the privacy of private network. But the private line is expensive, especially long distance line ${ }^{[2] .}$ The Virtual Private Network is the use of various authentication, encryption, detection technology to build a high degree of security data communication tunnel in the second and three layers. To use of VPN can transmit over the Internet and other high level enterprise confidential data ${ }^{[3]}$. Therefore, the networking scheme of VPN server based on the performance in terms of cost, reliability and, network information systems are much better than traditional.

\section{The Technology of VPN}

\subsection{The definition of VPN technology}

VPN technology is the network layer which used packet encapsulation technology and encryption technology, the packet can transmit in the public network which can "walk" in the "encryption pipeline". The public network to build their own "special" network security, it is an extension to the internal network. There many kinds of ways to identify verification, such as, the user name, password, the pre-shared key, certificates, smart card (hardware encryption) and IPSec. And it ensured the security of the data transmission and authentication in the whole process, when it encryption. VPN can help remote users, the company branches, business partners and suppliers with the company's internal network to establish credible security, and ensure the security of data transmission. The data transfer to the low cost of the network and virtual private network, this business solution will greatly reduce the user cost in the metropolitan area network and the remote network connection [4]. It will simplify the network design and management, and accelerate the connection of new users and web sites. In addition, the virtual private network can protect the existing network investment.

\subsection{The characteristics and classification}

VPN builds with high security on the data channel. It has the following characteristics: It can 
make sure the data authenticity. First, the communication host authorized have to address the fake (IP Spoofing) defensive ability, and make the data integrity guaranteed. Second, the receiving data sent with exactly the same, it has the ability to resist the illegal modification of data compilation, and makes sure the channel security guarantee. With dynamic key encryption technology and efficient exchange function, to ensure that the data channel is not illegal eavesdropper crack. It provides centralized security management services. Third, it has the means of security and access control, to defense the hackers by VPN channel threats to the corporate network implementation, and it can control implement access on VPN channel [5].

The application of VPN classification can be divided into two kinds. One is the virtual enterprise network. The other is a Virtual Private Dial-up Network (VPDN). This former mainly is the application of dedicated Internet company division, virtual private network between business partners. The latter refers to the telephone dial (PPP dial) virtual private network between Internet users and remote enterprise intranet [6].

\section{VPN Technology Network Construction Based on Enterprise Security}

\subsection{The requirements of the enterprise network system and security}

Enterprise information transmission network needs the high efficiency between the mutual communications. Mutual communication to ensure the security of data, and communication cost is low. Therefore, enterprises in the deployment of the transmission network, they need to consider its safety, adequate and real security. Whether the performance is sufficient meet the needs. It is convenient to deploy or not. You need to modify the existing network or not. Whether the existing network transformed and expands. The client configuration is simple or not. It can provide uninterrupted stability for the enterprise, stable and reliable connection or not. The strategy is flexible or not. When the user needs to change, it will quickly realize or not. When the user needs to change, or increase the number of concurrent user connections, the server can be expanded easily or difficult. The system is compatible with the industry standard or not. Whether the users use the various operating systems have good convergence.

\subsection{The application of VPN technology in the enterprise network security construction}

It considered the safety and economy based on analyzing the needs of corporation. This system is based on the third layer (network layer) of the IPsec VPN technology.

\subsubsection{VPN security mechanism}

VPN is not safe for communication in Internet. The content of the communication may involve confidential data of the enterprise. So its safety is very important. We must take a series of security mechanisms to ensure the safety of VPN. The security mechanism of VPN usually consists of encryption, authentication, key exchange and management. Conclusion based on the security architecture of IPsec. It is shown in the Figure 1.

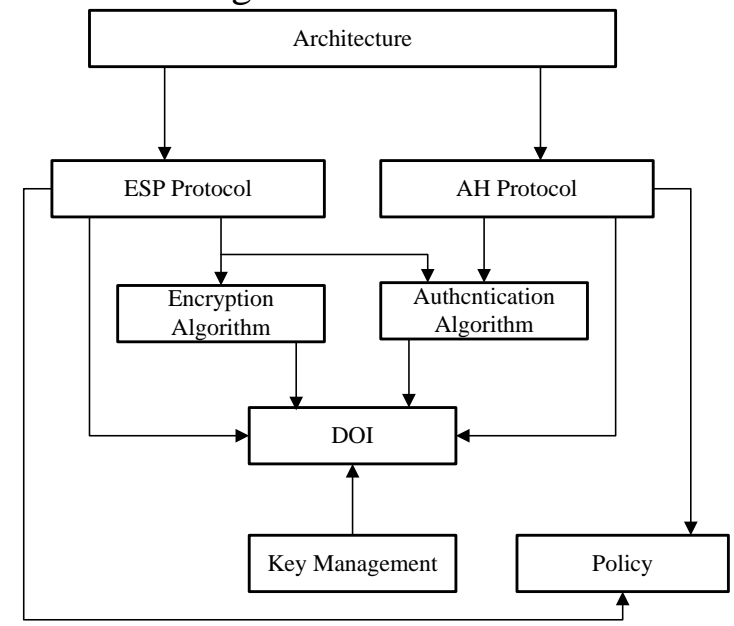

Figure 1. The architecture of IPsec 


\subsubsection{Design of construction enterprise security network based on VPN Technology}

A good VPN construction plan is a comprehensive enterprise information security solution. Its Comprehensive shows that it can fully protect the internal mutual communication, companies and strategic partners communicate with each other, communication between the two branches of the company and the company headquarters, and remote mobile staff communication with the company. The design scheme is shown in Figure 2.

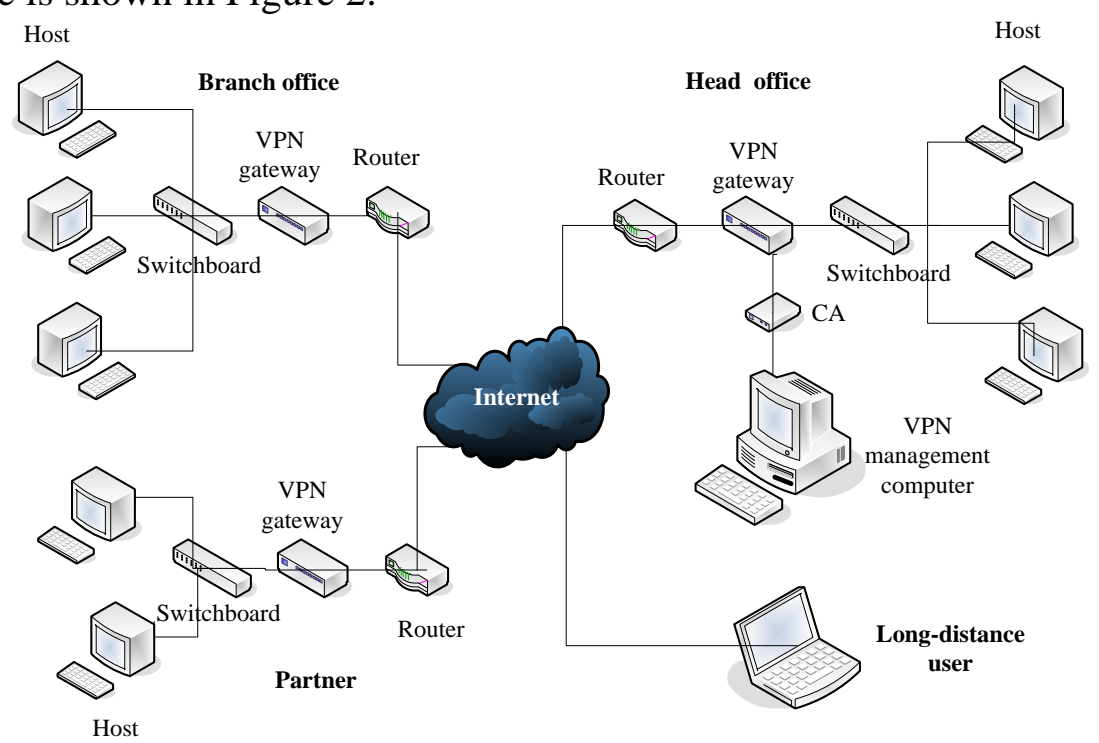

Figure 2. VPN construction

In the above figure, network environment includes a group headquarters LAN, multi branch LAN, and a plurality of connected with the strategic partner of Intranet. In addition, there are a number of foreign travel remote mobile users. The company internal network and branch network to establish a network to network tunnel which through VPN gateway. At the same time, the company internal network and the strategic partnership can establish a network to network tunnel. In the field staff can be accessed by dialing mobile company intranet, the data can be encrypted by the protection between VRC and company VPN gateway. In the network system, the full realization of the secure communication network to the network, end to communication network security.

\section{Conclusion}

Based on the analysis of the characteristics of VPN and the demand of the enterprise, the formation of enterprise network security by VPN, it not only saves the network construction and maintenance costs, and enhances network security and configuration flexibility. To encrypt and decrypt data packets by used VPN technology, and it implemented to the system. This is based on the third layer (network layer) of the IPsec VPN technology to conduct a comprehensive network construction. The problems of the traditional network constructed in the application layer end-to-end encryption management difficult, interoperability lower, system installation and migration are solved. VPN construction enterprise security network has low cost, strong adaptability, high standardization, convenient management, and with other security management technology and system integration technology integration easily. It can fully meet the needs of enterprises in the system and security.

\section{References}

[1] N Goyal, N Olver, FB Shepherd. The VPN Conjecture Is True [J]. Journal of the Acm, 2013, 60(3):443-450.

[2]Wang Haiping, Deng Wenwen. The Application of VPN Technology in the Collaborative Office in E-government [J]. Jiangsu Science and Technology Information, 2015(2):55-56.

[3]Xu Wei, Lou Songtao. Study on the Application of VPN Technology in Computer Network [J]. 
Electronic Technology and Software Engineering, 2014(4):239-240.

[4]Wang Bin, Gao Kun. Small Business Network Construction Based on VPN Technology [J]. Journal of Zhejiang Wanli University, 2015(4):85-90.

[5]Zhao Xiangmei, Du Xiaochun, Hou Yaling. The problems and Strategies of Small and Medium Sized Enterprises Network Security [J]. Electronic Test, 2014 (3X): 134-135.

[6]Li Hui. Enterprise Network Security Management [J]. Small and Medium-sized Enterprise Management and Technology Management, 2013 (7):256-256.

[7] Feng Haixiang. Research on Computer Network Security and Enterprise Network Security Applications [J]. Electronic Technology and Software Engineering, 2015(5):229-229.

[8] Lian Xiao. The Design and Practice of Enterprise Network Security [J]. Information System Engineering, 2014(7):72-72.

[9] Yang Min. Discussion on the Construction of Enterprise Information Security Management System [J].Electronic Technology and Software Engineering, 2013(24):238-238.

[10] Zhang Li.Virtual Private Network VPN Technology and Enterprise Application [J]. Chian New Telecommunication, 2013(3):59-59. 